$12-31-2016$

\title{
Leveraging the Windows Amcache.hve File in Forensic Investigations
}

\author{
Bhupendra Singh \\ Defence Institute of Advanced Technology (DU) \\ Upasna Singh \\ Defence Institute of Advanced Technology (DU)
}

Follow this and additional works at: https://commons.erau.edu/jdfs|

Part of the Computer Engineering Commons, Computer Law Commons, Electrical and Computer Engineering Commons, Forensic Science and Technology Commons, and the Information Security Commons

\section{Recommended Citation}

Singh, Bhupendra and Singh, Upasna (2016) "Leveraging the Windows Amcache.hve File in Forensic Investigations," Journal of Digital Forensics, Security and Law: Vol. 11 : No. 4 , Article 7. DOI: https://doi.org/10.15394/jdfsl.2016.1429 Available at: https://commons.erau.edu/jdfsl/vol11/iss4/7

This Article is brought to you for free and open access by

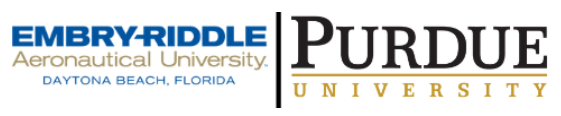
the Journals at Scholarly Commons. It has been accepted for inclusion in Journal of Digital Forensics, Security and Law by an authorized administrator of Scholarly Commons. For more information, please contact commons@erau.edu.

(c)ADFSL 


\title{
LEVERAGING THE WINDOWS AMCACHE.HVE FILE IN FORENSIC INVESTIGATIONS
}

\author{
Bhupendra Singh and Upasna Singh \\ Defence Institute of Advanced Technology (DU) \\ Girinagar, Pune - 411025 \\ \{bhupendra_pcse14, upasnasingh\}@diat.ac.in
}

\begin{abstract}
The Amcache.hve is a registry hive file that is created by Microsoft ${ }^{\circledR}$ Windows ${ }^{\circledR}$ to store the information related to execution of programs. This paper highlights the evidential potential of Amcache.hve file and its application in the area of user activity analysis. The study uncovers numerous artifacts retained in Amcache.hve file when a user performs certain actions such as running host-based applications, installation of new applications, or running portable applications from external devices. The results of experiments demonstrate that Amcache.hve file stores intriguing artifacts related to applications such as timestamps of creation and last modification of any application; name, description, publisher name and version of applications; execution file path, SHA-1 hash of executable files etc. These artifacts are found to persist even after the applications have been deleted from the system. Further experiments were conducted to evaluate forensic usefulness of the information stored in Amcache.hve and it was found that Amcache.hve information is propitious to trace the deleted applications, malware programs and applications run from external devices. Finally, comparison of information in Amcache.hve file with information in other similar sources (IconCache.db, SRUDB.dat and Prefetch files) is shown, in order to provide more useful information to forensic investigators.
\end{abstract}

Keywords: Amcache.hve, Windows Registry Forensics, User Activity Analysis, Program Execution Analysis, Malware Analysis

\section{INTRODUCTION}

In digital forensic investigations, it is sometimes essential to carry out user activity analysis on a suspected system to know recently accessed file and folders, run applications and their execution history. User activity analysis is also helpful in detection of the anti-forensic behaviors carried out to hinder investigations. The Amcache.hve file is a very useful resource in the area of user activity analysis to analyze recently run applications and their execution history. Earlier, the forensic analysts had access to RecentFileCache.bcf file, which has been replaced by Amcache.hve file in Windows 8 and later versions. Harrell (2013) has highlighted the forensic artifacts stored in RecentFileCache.bcf file. However, the Amcache.hve being newly introduced, is not yet 
explored in digital forensics community.

The Amcache.hve stores information related to Windows Application Experience and Compatibility feature in a registry hive file. Forensic analysis of Amcache.hve file can reveal important artifacts such as program name and version, execution file path, file size, installation timestamp, the timestamp of first run, hash of executable file etc. This information persists in Amcache.hve even when the applications and Prefetch folder have been deleted on a user's computer. Therefore, Amcache.hve is also useful in detection of anti-forensic tools, thus, revealing the intentions of a user. Although, the Amcache.hve exposes forensically useful information, it has not been leveraged by forensic analysts as an analytical method for detection of anti-forensic behaviors.

The traces of recently run applications and their usefulness in forensic investigations have been discussed in various literature published. Singh \& Singh (2016) have shown the traces of recently accessed files and run applications in Jump Lists on a Windows system. The authors demonstrated that the traces of run applications persist even after the applications are removed from the system. Carvey $(2005,2011)$ and Wong (2007) have described that numerous artifacts related to run programs on Windows system are recorded in UserAssist registry key. Mee \& Jones (2005), Carvey \& Altheide (2005) and Mee et al. (2006) have investigated the UserAssist key to identify the external devices that have been connected to the system in order to run a malware program or an anti-forensic tool. Collie (2013) has examined the evidential potential of Windows IconCache.db file and found that IconCache.db file stores artifacts related to executable files when they are present on or run from USB connectable devices. Further, Lee \& Lee (2014) identified the structure and highlighted the importance of Icon-
Cache.db file in forensic investigations. The authors demonstrated that IconCache.db file stores full file paths for executable files that have been run, viewed, or installed on a Windows system. Khatri (2015) has examined the SRUDB.dat file in Windows 8 as a new database for tracking user activities and linking launched programs by users. The author has demonstrated the usefulness of SRUDB.dat database in forensic investigations by tracking Windows Store App runs, tracking processes run from external media devices, tracing deleted processes and antiforensic tools. In recent study, Singh \& Singh (2017) provides in-depth understanding of the artifacts and their location created by Cortana application in Windows 10 system. However, we could not find any literature published on forensic analysis of Amcache.hve file except a brief narration by Kim \& Lee (2015).

This study mainly investigates the artifacts created and retained in the Amcache.hve file when a user performs certain actions, such as running executable files from host or from USB devices. The effects on Amcache.hve of installing package applications containing many executables files tied into a package, are also considered. The paper provides an overview of how the Amcache.hve file comes into existence and how it grows in size and records the artifacts. The effects of anti-forensic attempts on Amcache.hve are also taken into consideration, however, results of this experiment may vary depending upon the version of Windows operating system. Furthermore, the forensic application of Amcache.hve is evaluated in terms of tracking deleted applications, malware programs and applications run from external storage devices. The paper also identifies the locations and artifacts recorded in interrelated files, however, the prime goal of study is to extract the information retained in Amcache.hve file and its application in 
digital forensics.

\section{MOTIVATION AND RESEARCH METHODOLOGY}

There exists few posts on digital forensic blogs which describe the forensic usefulness of Windows 8 Amcache.hve file. However, the effect of user activities on Amcache.hve file in different distributions of Windows is not yet highlighted in forensic community.

The research method used in this paper is based on observations and experiments. For studying the behavior of Amcache.hve with respect to user activities, several experiments were conducted on three test computers: a laptop running Windows 8 Pro, a desktop running Windows 8.1 Pro, and another laptop running Windows 10 Pro (v1511). A number of user activities were carried out on these test computers in accordance with the objective of the experiment. For extracting Amcache.hve file from test computers, a Live Boot DVD of Ubuntu 14.04 LTS was used. The Amcache.hve file from each test computer was then parsed using Registry Explorer (Zimmerman, 2015). The findings drawn on the basis of observations are discussed in Section 4. Apart from it, Amcache.hve files from several other systems running different distributions of Windows were extracted and analyzed to validate the findings.

\section{AMCACHE.HVE FILE IN WINDOWS}

Microsoft Windows operating systems use Application Experience and Compatibility feature to ensure the compatibility of software among different distributions of Windows operating system (Microsoft, 2016). For this, Microsoft implements Windows
Compatibility Infrastructure (Shim Infrastructure) technique in Windows operating systems. The mechanism of Shim Infrastructure technique is beyond the scope of this research. However, the implementation of this feature generates some interesting artifacts related to program executions that can be leveraged in digital forensic investigations. Davis (2012) leveraged application compatibility cache data to recover artifacts such as file size, file last modified time and last execution time. In this research, we highlight the usefulness of Amcache.hve file in forensic investigations as one of the artifacts associated with Application Experience and Compatibility feature.

The Amcache.hve is a registry hive file introduced in Windows 8 as the replacement of Windows 7 RecentFileCache.bcf file under \%SystemDrive\% $\backslash$ Windows $\backslash$ App Compat $\backslash$ Programs directory. We observed that the Amcache.hve is now also available in Windows 7 systems since October 2015. Depending upon the operating system in use, the Amcache.hve file is stored at following locations:

- Windows 7/8/8.1: \%SystemDrive $\% \backslash$ Windows $\backslash$ AppCompat $\backslash$ Programs $\backslash$ Amcache.hve

- Windows 10: \%SystemDrive\% \Windows $\backslash$ appcompat $\backslash$ Programs $\backslash$ Amcache.hve

The structure of Amcache.hve file follows Windows NT Registry File (REGF) format. Microsoft uses REGF format in Windows NT (or later) to store a part of the Windows registry. There are many software tools (Carvey, 2013; NirSoft, 2013; AccessData, 2014; Zimmerman, 2015) available to parse and view the registry file. The following subsections describe the information recorded in Amcache.hve and its interrelated files. 


\subsection{The Hive}

The Amcache.hve file contains sections of 4KB referred as "bins." As the Amcache.hve file grows, these bins make allocation as well as maintenance of the hive file itself. The Amcache.hve starts with four bytes signature value "regf" (0x72656766). Figure 1 shows the hierarchical structure of Amcache.hve file; seven subkeys (folders) under the 'Root' key were observed. The information of interest to a forensic analyst is located in the 'File' and the 'Programs' subkeys. The subkeys under the File key are the Volume Globally Unique Identifiers (GUIDs) of the mounted devices. The information related to mounted devices can be corroborated with the values of registry key at HKLM $\backslash \mathrm{SYSTEM} \backslash$ MountedDevices.

\section{$3.2 \quad$ File References}

Each Volume GUID stores file reference keys and each file reference key represents a unique file on disk (Khatri, 2013). The values of the file reference keys were observed from 3 to 23 in Windows 8/8.1/10. Table 1 shows value name, value type and data for file reference keys. Minimum three values were observed under any file reference key namely 15,17 and 100 . The value 15 stores full path to the executable file from that was run. The value 100 represents the program ID that may be found under the Programs key. The value 101 represents the SHA-1 hash of the executable file preceded by four extra 0s. The last write timestamp on a reference key in Registry Explorer is considered as the timestamp of application's first run. No change was observed in timestamp on subsequent runs of applications. Few file reference keys store all 23 values, thus, containing more information related to that executable file. The value 11 and 17 represent last modified timestamps which were observed to be same when decoded. Major portion of file reference keys was identified; however, the purpose of few fields (data in values $a, b, d, 10)$ remains unknown.

Table 1. Stored information in file reference keys

\begin{tabular}{|c|c|c|}
\hline Value & Value type & Description \\
\hline 0 & Unicode & Program name \\
\hline 1 & Unicode & Publisher name \\
\hline 2 & Unicode & Program version (major) \\
\hline 3 & DWORD & Language Code \\
\hline 4 & QWORD & SwitchBackContext \\
\hline 5 & Unicode & Program version (minor) \\
\hline 6 & DWORD & File size (in bytes) \\
\hline 7 & DWORD & $\begin{array}{l}\text { PE Header field- } \\
\text { SizeOfImage }\end{array}$ \\
\hline 8 & Unicode & Hash of PE Header \\
\hline 9 & DWORD & $\begin{array}{l}\text { PE Header field- } \\
\text { Checksum }\end{array}$ \\
\hline 10 & DWORD & $\begin{array}{l}\text { Unknown (always } 0,6 \\
\text { or } 13 \text { in all tests) }\end{array}$ \\
\hline 11 & FILETIME & Last modified timestamp \\
\hline 12 & FILETIME & Created timestamp \\
\hline 15 & Unicode & Full path to file \\
\hline 16 & DWORD & $\begin{array}{l}\text { Unknown ( } 0 \text { or } 1 \text { for all } \\
\text { tests) }\end{array}$ \\
\hline 17 & FILETIME & $\begin{array}{l}\text { Last modified } \\
\text { timestamp } 2\end{array}$ \\
\hline $\mathrm{a}$ & QWORD & Unknown \\
\hline $\mathrm{b}$ & QWORD & Unknown \\
\hline $\mathrm{c}$ & Unicode & File description \\
\hline $\mathrm{d}$ & DWORD & Unknown \\
\hline $\mathrm{f}$ & DWORD & Linker timestamp (Unix) \\
\hline 100 & Unicode & Program ID \\
\hline 101 & Unicode & $\begin{array}{l}\text { SHA-1 hash of executable } \\
\text { file }\end{array}$ \\
\hline
\end{tabular}

\subsection{Programs Key}

The subkeys under the Programs key are the program IDs assigned to each MSI package. The information stored in a program ID is similar to the information in a file reference key. The number of values in a program ID was observed from 12 to 21 in Windows 8/8.1 


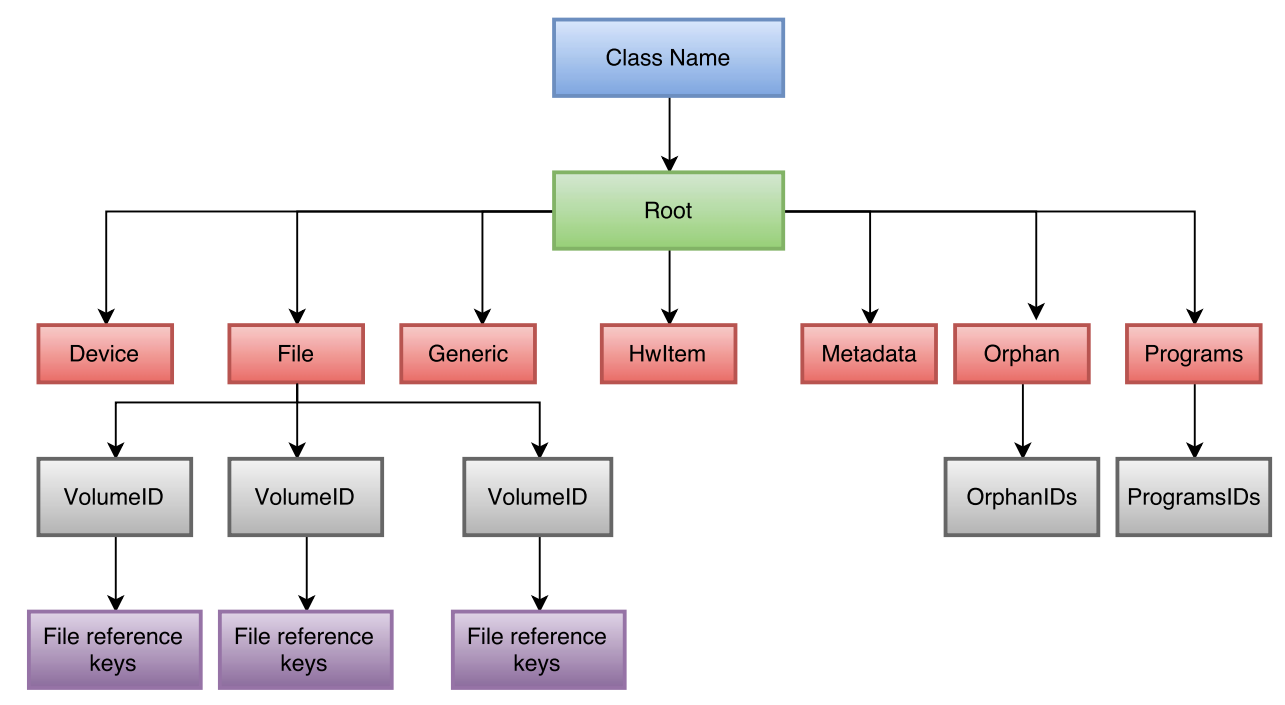

Figure 1. Directory structure of Amcache.hve file

and 16 to 21 in Windows 10 system. Each program ID contains important information related to installed application as summarized in Table 2. For all the cases, the data in value 10 and 12 represents package GUID and was found to be same. Further, the data in value 11 and $f$ describes product GUID and was also observed to be same. The product GUID was also found in uninstall registry key (value 7). The information in value $a$ represents the timestamp of application's installation and the information in value $b$ represents the timestamp when the application was deleted (uninstalled). In the analysis, data in value Files was found as list of files referenced by package applications. Each referenced file under Files list is represented as VolumeGUID@FileRef where FileRef is the file reference key beneath Volume GUID of the File key. In testing, it was observed that not all referenced files in Files list could be found in file reference keys. The relevant fields in a program ID were identified; however, purpose of few fields remains unknown that can be explored in further study.

\subsection{Inter-related Files}

It was observed that there exists four inventory files associated with Amcache.hve file; however, these files are temporarily stored on disk and automatically created and deleted during user actions. The location of three inventory files: ApplicationInventory.xml, DeviceInventory.xml and FileInventory.xml is under the directory \%SystemDrive\%\Windows $\backslash$ appcompat $\backslash$ appraiser $\backslash$ Gated in Windows 10. Apart from these three inventory files, one more inventory file associated with Amcache.hve file was observed under the directory \%SystemDrive\%\Windows $\backslash$ appcompat $\backslash$ Programs. This inventory file consists information related to installed programs, files and Internet Explorer Add-on list.

\section{EXPERIMENTS AND RESULTS}

The experiments are divided in three sets: baseline Amcache.hve research (Set A), advanced Amcache.hve research (Set B) and anti-forensic attempts on Amcache.hve (Set C). In Set A, the baseline behavior of Am- 
Table 2. Stored information in programs ID keys

\begin{tabular}{|c|c|c|}
\hline Value & Value type & Description \\
\hline 0 & Unicode & Program name \\
\hline 1 & Unicode & Program version \\
\hline 2 & Unicode & Publisher name \\
\hline 3 & Unicode & Language Code \\
\hline 5 & DWORD & Unknown \\
\hline 6 & Unicode & $\begin{array}{l}\text { Install source (always Msi, } \\
\text { AddRemoveProgram or } \\
\text { AddRemoveProgramPerUser } \\
\text { in all tests) }\end{array}$ \\
\hline 7 & Unicode & Uninstall Registry key \\
\hline 10 & Unicode & Package GUID \\
\hline 11 & Unicode & MSI product GUID \\
\hline 12 & Unicode & MSI package GUID \\
\hline 13 & DWORD & $\begin{array}{l}\text { Unknown (always } 0 \text { in } \\
\text { all tests) }\end{array}$ \\
\hline 14 & DWORD & $\begin{array}{l}\text { Unknown (always } 0 \text { in } \\
\text { all tests) }\end{array}$ \\
\hline 15 & DWORD & $\begin{array}{l}\text { Unknown (always } 0 \text { in } \\
\text { all tests) }\end{array}$ \\
\hline 16 & Binary & Unknown \\
\hline 17 & QWORD & $\begin{array}{l}\text { Unknown (always } 28147497 \\
67116800 \text { in all tests) }\end{array}$ \\
\hline 18 & DWORD & $\begin{array}{l}\text { Unknown (always } 0 \\
\text { or } 1 \text { in all tests) }\end{array}$ \\
\hline $\mathrm{a}$ & QWORD & Install timestamp (Unix) \\
\hline $\mathrm{b}$ & QWORD & $\begin{array}{l}\text { Uninstall timestamp (Unix, } \\
0 \text { if program is not uninstalle }\end{array}$ \\
\hline $\mathrm{d}$ & Unicode & List of file path \\
\hline$f$ & Unicode & Product GUID \\
\hline Files & Unicode & $\begin{array}{l}\text { List of file entries in package } \\
\text { (VolumeGUID@FileRef) }\end{array}$ \\
\hline
\end{tabular}

3. The number of default applications present in Amcache.hve file depends upon the operating system under consideration and may vary because of the configuration settings selected at the time of installation. Table 5 shows the default applications installed during a clean installation of different Windows operating systems.

4. First reboot of the system results in creation of 'Application 
Table 3. Details of experiments devised

\begin{tabular}{|c|c|c|}
\hline Set & Objective & Actions \\
\hline $\begin{array}{l}\text { A: Baseline } \\
\text { Amcache.hve research }\end{array}$ & Establish file provenance & $\begin{array}{l}\text { To ascertain: } \\
\text { 1. When the Amcache.hve is created. } \\
\text { 2. Its content just after creation. } \\
\text { 3. Interrelated files and their content. } \\
\text { 4. Amcache.hve content following the system reboot or } \\
\text { shutdown. }\end{array}$ \\
\hline $\begin{array}{l}\text { B: Advanced } \\
\text { Amcache.hve } \\
\text { research }\end{array}$ & $\begin{array}{l}\text { To observe the content of } \\
\text { Amcache.hve file and } \\
\text { interrelated files after repeated } \\
\text { user activities }\end{array}$ & \\
\hline B1 & $\begin{array}{l}\text { To observe the effects of } \\
\text { basic user activities }\end{array}$ & $\begin{array}{l}\text { To ascertain: } \\
\text { 1. Amcache.hve content after running a host-based executable. } \\
\text { 2. Content of interrelated files after running a host-based } \\
\text { executable }\end{array}$ \\
\hline B2 & $\begin{array}{l}\text { To observe the effects of } \\
\text { installing an application from host }\end{array}$ & $\begin{array}{l}\text { To ascertain: } \\
\text { 1. Content of Amcache.hve file after installing an application. } \\
\text { 2. Content of interrelated files after installing an application. } \\
\text { To ascertain: }\end{array}$ \\
\hline B3 & $\begin{array}{l}\text { To observe the effects of } \\
\text { connecting external devices }\end{array}$ & $\begin{array}{l}\text { 1. Content of Amcache.hve file after an executable file is } \\
\text { run from a USB drive. } \\
\text { 2. Content of Amcache.hve after an executable file is } \\
\text { installed from a USB drive. } \\
\text { To ascertain: }\end{array}$ \\
\hline B4 & $\begin{array}{l}\text { To observe the effects of } \\
\text { Metro Apps }\end{array}$ & $\begin{array}{l}\text { 1. Content of Amcache.hve after installing a } \\
\text { Metro App. } \\
\text { 2. Content of interrelated files after installing } \\
\text { a Metro App. }\end{array}$ \\
\hline $\begin{array}{l}\mathrm{C}: \text { Anti-forensic } \\
\text { attempts }\end{array}$ & $\begin{array}{l}\text { To observe the effects of } \\
\text { anti-forensic attempts }\end{array}$ & $\begin{array}{l}\text { To ascertain: } \\
\text { 1. What happens if the Amcache.hve file is deleted. } \\
\text { 2. What happens when interrelated files are deleted. } \\
\text { 3. Modifying the timestamps of Amcache.hve. }\end{array}$ \\
\hline
\end{tabular}

Table 4. Software tools used in study

\begin{tabular}{ll}
\hline Software tool & Purpose \\
\hline Registry Explorer & To parse Amcache.hve file \\
DCode & Binary data to date/time converter \\
HxD Hex Editor & To view/modify raw content of Amcache.hve file \\
\hline a Available from: $\quad$ http://www.digital-detective.net/digital \\
-forensic-software/free-tools/ \\
b Available from: https://mh-nexus.de/en/hxd/
\end{tabular}

Experience Inventory' file at the system. same location of Amcache.hve file. The inventory file is named as AEINV_AMI_WER_\{MachineID $\}$ DATE _TIME.xml, where DATE and TIME are file creation date and time (in GMT) while MachineID is 16 bytes GUID value generated by Windows

5. It was observed that no other associated file(s) was created until no user activity was performed except system shutdown or reboot. 
Table 5. Applications in Amcache.hve on initial creation

\begin{tabular}{|c|c|c|c|}
\hline & Windows 8 Pro (Build 9200) & Windows 8.1 Pro (Build 9600) & Windows 10 Pro (Build 10240) \\
\hline $\begin{array}{l}\text { Size of } \\
\text { Amcache.hve } \\
\text { (in KB) }\end{array}$ & $\begin{array}{l}\mathrm{C}: \backslash \text { Windows } \backslash \text { System } 32 \backslash \text { csrss.exe } \\
\mathrm{C}: \backslash \text { Windows } \backslash \text { System } 32 \backslash \text { dwm.exe } \\
\mathrm{C}: \backslash \text { Windows } \backslash \text { System } 32 \backslash \text { LogonUI.exe } \\
\mathrm{C}: \backslash \text { Windows } \backslash \text { System } 32 \backslash \text { services.exe } \\
\mathrm{C}: \backslash \text { Windows } \backslash \text { System } 32 \backslash \text { smss.exe } \\
\mathrm{C}: \backslash \text { Windows } \backslash \text { System } 32 \backslash \text { SppExtComObj.Exe } \\
\mathrm{C}: \backslash \text { Windows } \backslash \text { System } 32 \backslash \text { taskhost.exe } \\
\mathrm{C}: \backslash \text { Windows } \backslash \text { System } 32 \backslash \text { taskhostex.exe } \\
\mathrm{C}: \backslash \text { Windows } \backslash \text { System } 32 \backslash \text { wininit.exe } \\
\mathrm{C}: \backslash \text { Windows } \backslash \text { System } 32 \backslash \text { winlogon.exe } \\
\mathrm{C}: \backslash \text { Windows } \backslash \text { System } 32 \backslash \text { oobe } \backslash \text { msoobe.exe } \\
\mathrm{C}: \backslash \text { Windows } \backslash \text { System } 32 \backslash \text { wbem } \backslash \text { WmiPrvSE.exe } \\
\mathrm{C}: \backslash \text { Windows } \backslash \text { SysWOW64\ByteCodeGenerator.exe }\end{array}$ & 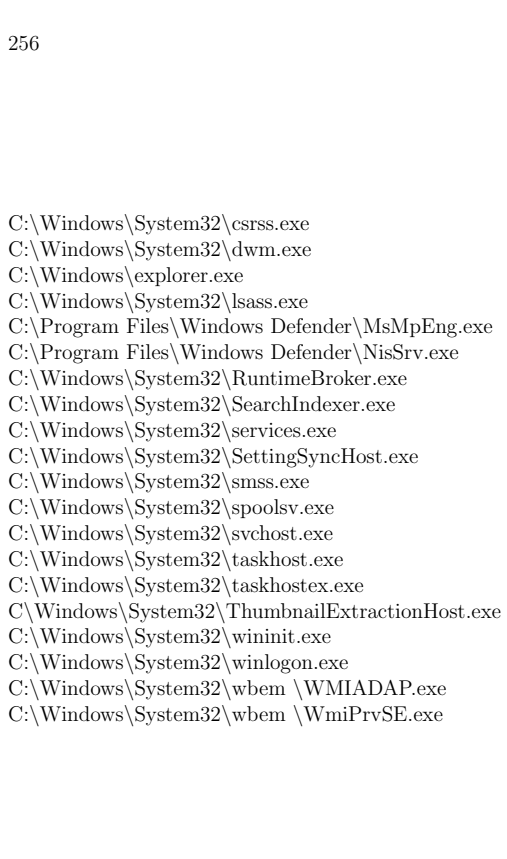 & 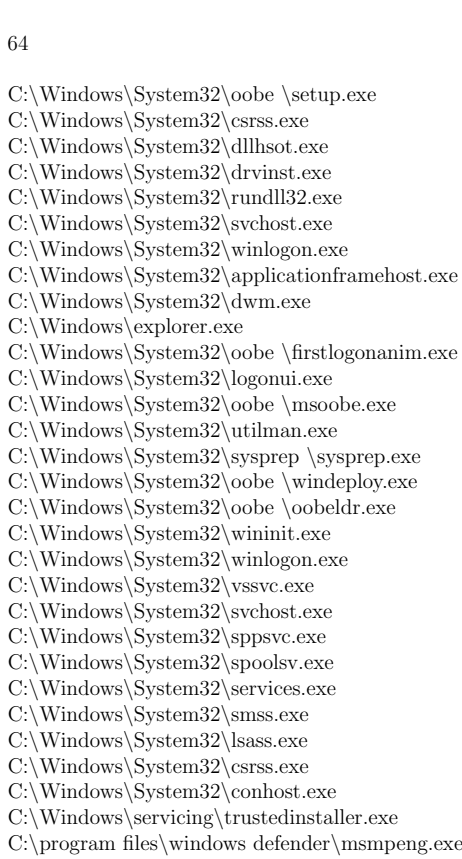 \\
\hline
\end{tabular}

\subsection{Set B: Advanced Amcache.hve Research}

Reconstructing the artifacts left by user activities on a computer system is part of many forensic examinations where user activity is of investigators' interest. The following subsections describe the behavior of Amcache.hve and its interrelated files when different user activities are performed on a Windows system. For this, Windows 10 Pro system was taken into consideration and the numerous user activities were carried out including running host-based executable programs, installing of software applications, running an executable file from a USB thumb drive, visiting URLs from web browsers, installing Metro Apps from Windows Store etc. The effects of such user activities on Amcache.hve were observed and the findings are summarized in the next subsections.

\subsubsection{B1: Running a host-based executable}

It was observed that when a host-based executable is run for the first time after installation of operating system, the timestamp of run along with executable file path are recorded in Amcache.hve. No artifacts related to run count is recorded in Amcache.hve file and its interrelated files. However, the timestamp of last modification is recorded in Amcache.hve file, which is always the timestamp of first time the executable was run. It means that successive runs of the host-based executable does not effect the Amcache.hve file. Figure 2 shows the timestamp of first run and executable file path of notepad application.

\subsubsection{B2: Installing a software program from host}

For conducting this experiment, Mozilla Firefox was installed from the host and subsequently run for the first time. The Am- 


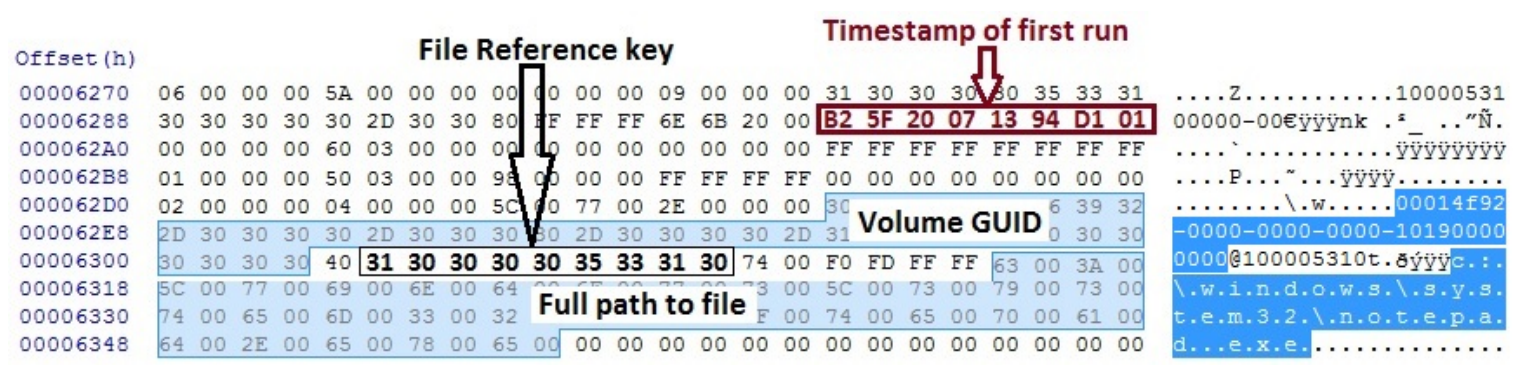

Figure 2. Timestamp of first run and executable file path of notepad application recorded in Amcache.hve

cache.hve file is extracted and then analyzed using HxD hex editor and the artifacts related to Mozilla Firefox were identified. We observed that when a software program is installed then there are two locations in Amcache.hve file at which related artifacts are found. At first location, the full file path from which the executable was run is recorded in file reference key under File key. Figure 3 shows the full file path of executable of Mozilla Firefox from where it was run along with the timestamp of run. At second location in program ID of Mozilla Firefox under the Programs key recorded numerous artifacts such as program name, program version, publisher name, program type etc.

\subsubsection{B3: Installing and running a software program from external storage media}

This experiment was performed to know the type of artifacts retained in Amcache.hve file when the applications are installed from external devices. A package application (Proxifier Version 3.21 for our case) containing five executables tied into a package was installed from a USB drive to test whether information related to all executables is recorded. Subsequently, the system was allowed to shutdown so that the modifications in the Amcache.hve file could be stored on disk. The Amcache.hve file was then extracted and analyzed using a hex editor. We found program ID, program name and version, install source, file referenced keys, uninstall registry key and full path to file as shown in Figure 4. The related information such as install timestamp, publisher name, package GUID and volume GUID were also observed in Amcache.hve file which are not shown in the figure for visual convenience. We found five file referenced keys namely: 200014f20, 200014f $13,200014 f 1 d, 200014 f 22$ and $600014 f 16$. Each file referenced key corresponds to a referenced file by the package application. For our case, 200014f20 referred to Proxifier.exe, $200014 f 13$ referred to ProxyChecker.exe, 200014f1d referred to SysSettings32.exe, $200014 f 22$ referred to SysSettings64.exe and 600014f 16 referred to unins000.exe. In addition to this, we also found the created timestamp of each referenced file with their file path in the Amcache.hve file.

The information related to referenced files by a package application can be very useful to forensic/malware analysts. The analysis of Amcache.hve file can provide the name of all referenced files by a malicious program.

When a portable program is run from USB drive, the artifacts of such portable programs are recorded in Amcache.hve file. Figure 5 shows various artifacts related to DCode program which was run from a USB drive, including timestamp of run, program name etc. It was observed that no information related to USB Volume label is recorded in Amcache.hve file; however, Amcache.hve file 


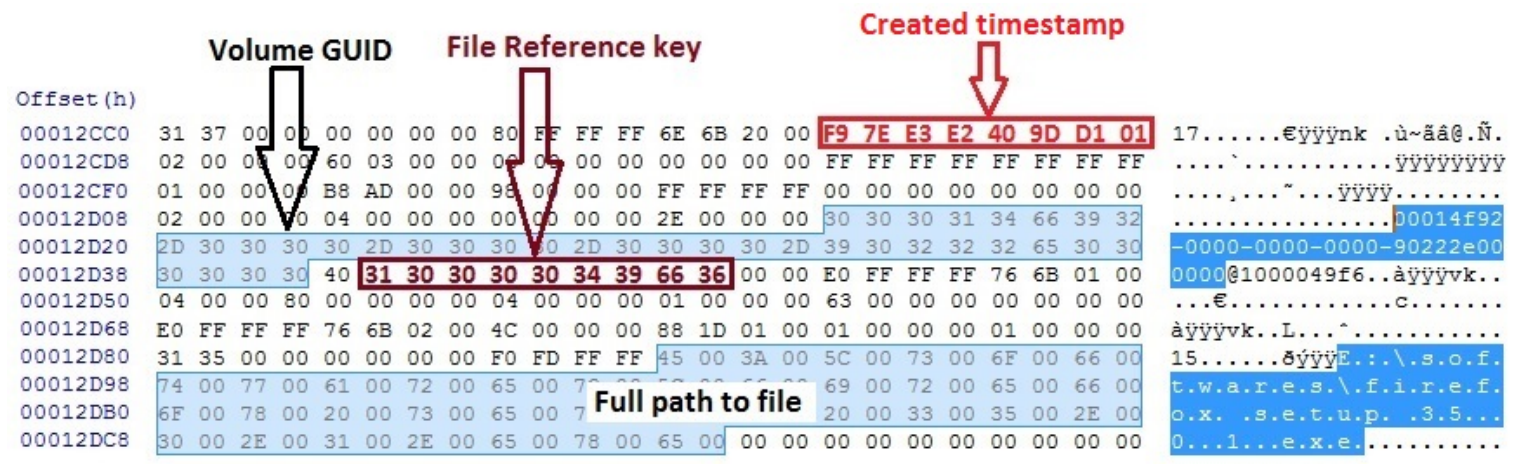

Figure 3. Timestamp of run and installed file path of Mozilla Firefox in Amcache.hve

$$
\begin{aligned}
& \text { Offset (h) } \\
& 00105 \mathrm{E} 88 \\
& 00105 \mathrm{EAO} \\
& 00105 \mathrm{~EB} 8 \\
& 00105 \mathrm{EDO} \\
& 00105 \mathrm{EE} 8 \\
& 00105 \mathrm{FFO} \\
& 00106008 \\
& \vdots \\
& 00106758 \\
& 00106770 \\
& 00106788 \\
& 001067 \mathrm{~A} 0 \\
& 001067 \mathrm{BB} \\
& 001067 \mathrm{DO} \\
& 001067 \mathrm{E} 8 \\
& 00106800 \\
& 00106818 \\
& 00106830 \\
& 00106848 \\
& 00106860 \\
& 00106878 \\
& 00106890 \\
& 001068 \mathrm{AB} \\
& 001068 \mathrm{CO} \\
& 001068 \mathrm{D} 8 \\
& 001068 \mathrm{~F} 0 \\
& 00106908 \\
& 00106920 \\
& 00106938 \\
& 00106950 \\
& 00106968 \\
& 00106980 \\
& 00106998 \\
& 001069 \mathrm{~B} 0 \\
& 001069 \mathrm{CB} \\
& 001069 \mathrm{E} 0 \\
& 001069 \mathrm{~F} 8 \\
& 00106 \mathrm{~A} 10 \\
& 00106 \mathrm{~A} 28 \\
& 00106 \mathrm{~A} 40 \\
& 00106 \mathrm{~A} 58 \\
& 00106 \mathrm{~A} 70 \\
& 0010 \mathrm{~A} 88 \\
& 0
\end{aligned}
$$

00106AA0

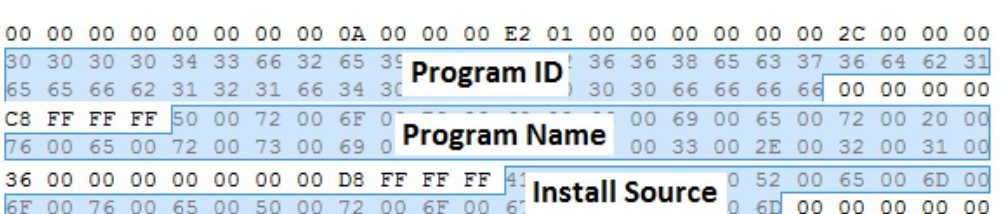

\section{File Referenced keys}

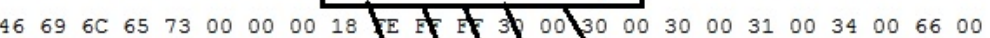

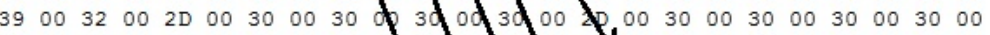

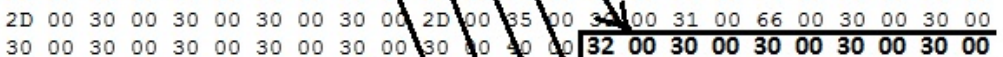
31003400660032003000 of 340003000310034006600

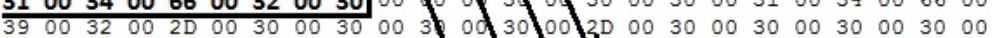

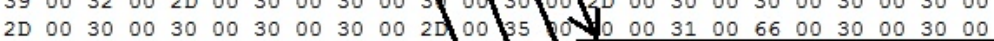

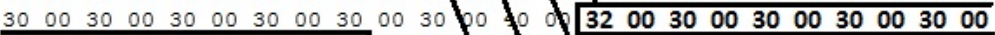

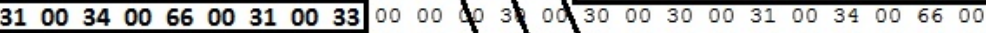
$\begin{array}{lllllllllllllllllllllllll}39 & 00 & 32 & 00 & 2 \mathrm{D} & 00 & 30 & 00 & 30 & 00 & 30 & 0 & 30 & 00 & 00 & 30 & 00 & 30 & 00 & 30 & 00 & 30 & 00\end{array}$

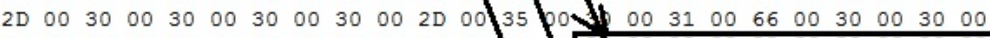
$\begin{array}{lllllllllllll}30 & 00 & 30 & 00 & 30 & 00 & 30 & 00 & 30 & 00 & 30 & 00 & 0\end{array}$

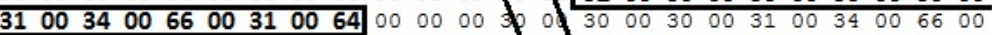
$\begin{array}{lllllllllllll}39 & 00 & 32 & 00 & 2 \mathrm{D} & 00 & 30 & 00 & 30 & 00 & 30 & 00 & 3\end{array}$

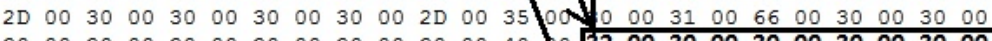

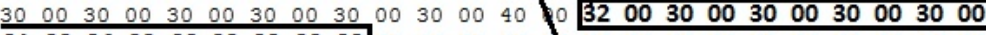

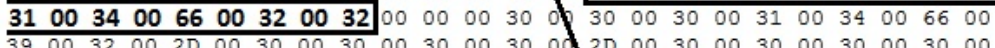
$\begin{array}{llllllllllllllll}39 & 00 & 32 & 00 & 2 \mathrm{D} & 00 & 30 & 00 & 30 & 00 & 30 & 00 & 30 & 0\end{array}$

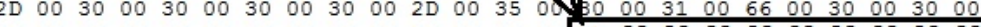
$\begin{array}{lllllllllllllllllllllllll}30 & 00 & 30 & 00 & 30 & 00 & 30 & 00 & 30 & 00 & 30 & 00 & 40 & 00 & 36 & 00 & 30 & 00 & 30 & 00 & 30 & 00 & 30 & 00\end{array}$

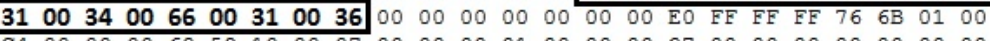

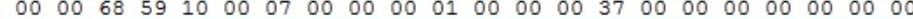
$\begin{array}{lllllllllllllllllllllllll}38 & \text { FF } & F F & F F & 48 & 00 & 4 \mathrm{~B} & 00 & 45 & 00 & 59 & 00 & 5 \mathrm{~F} & 00 & 4 \mathrm{C} & 00 & 4 \mathrm{~F} & 00 & 43 & 00 & 41 & 00 & 4 \mathrm{C} & 00 \\ 5 \mathrm{~F} & 00 & 4 \mathrm{D} & 00 & 41 & 00 & 43 & 00 & 48 & 00 & 49 & 00 & 4 \mathrm{E} & 00 & 45 & 00 & 5 \mathrm{C} & 00 & 53 & 00 & 6 \mathrm{~F} & 00 & 66 & 00\end{array}$ $\begin{array}{llllllllllllllllllllllll}5 \mathrm{~F} & 00 & 4 \mathrm{D} & 00 & 41 & 00 & 43 & 00 & 48 & 00 & 49 & 00 & 4 \mathrm{E} & 00 & 45 & 00 & 5 \mathrm{C} & 00 & 53 & 00 & 6 \mathrm{~F} & 00 & 66 & 00 \\ 74 & 00 & 77 & 00 & 61 & 00 & 72 & 00 & 65 & 00 & 5 \mathrm{C} & 00 & 57 & 00 & 6 \mathrm{~F} & 00 & 77 & 00 & 36 & 00 & 34 & 00 & 33 & 00\end{array}$ $\begin{array}{llllllllll}32 & 00 & 4 \mathrm{E} & 00 & 6 \mathrm{~F} & 00 & 64 & 00 \\ 6 \mathrm{~F} & 00 & 66 & 00 & 74 & 00 & 5 \mathrm{C} & 00\end{array}$ Uninstall Registry key

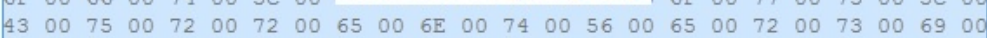

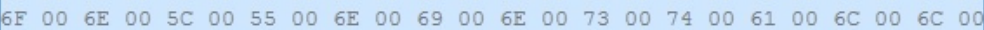

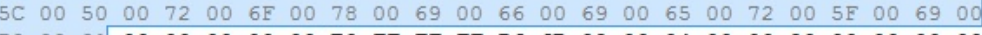
$\begin{array}{llllllllllllllllllllllllll}04 & 00 & 00 & 00 & 01 & 00 & 10 & 00 & 31 & 34 & 10 & 00 & \text { D8 } & 4 F & 10 & 00 & \text { FO } & \text { FF FF FF } & 00 & 00 & 00 & 00\end{array}$

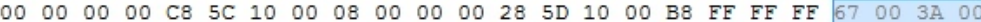

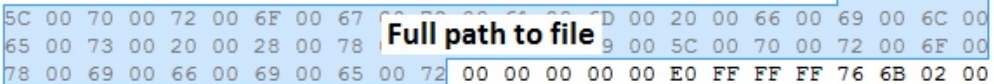

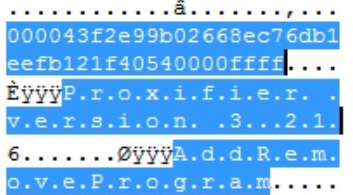

Files....pÿÿ0.0.0.1.4.f. $9.2 .-0.0 .0 .0 .-0.0 .0 .0$. $-0.0 \cdot 0 \cdot 0 .-.5 \cdot 0.1 .1 .0 .0$ $0.0 .0 .0 .0 .0 .0 .2 \cdot 0 \cdot 0.0 .0$ 1.4.f.2.0..0.0.0.1.4.f 9.2.-.0.0.0.0.-.0.0.0.0 $-.0 .0 .0 .0 .-.5 .0 .1 . f .0 .0$. $0.0 .0 .0 .0 .0 .0 .2 \cdot 0.0 .0 .0$ 1.4.f.1.3..0.0.0.1.4.f 9.2.-.0.0.0.0.- $0 \cdot 0 \cdot 0 \cdot 0$ 0.0.0.0.-.5.0.1.f.0.0. $0.0 .0 .0 .0 .0 .2 \cdot 0.0 .0 .0$ 1.4.f.1.d..0.0.0.1.4.f. 9.2.-.0.0.0.0.-.0.0.0.0. $-.0 .0 .0 .0 .-.5 .0 .1 . f .0 .0$ 0.0 .0 .0 .0 .0 .0 .2 .0 .0 .0 .0 . 1.4.f.2.2..0.0.0.1.4.f 9.2.-.0.0.0.0.-.0.0.0.0. $-0.0 .0 .0 .-.5 .0 .1 . f .0 .0$ 0.0 .0 .0 .0 .0 .0 .6 .0 .0 .0 .0 $1.4 . f .1 .6 \ldots \ldots$ ày̆y̆ÿvk.

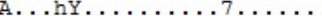

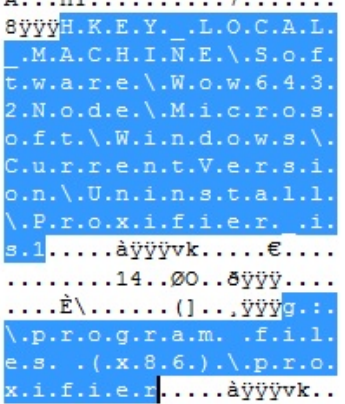

Figure 4. Recorded information related to application installed from USB drive

stores the drive letter assigned to a USB drive. Apart from it, Amcache.hve also stores Volume GUID of USB and the full path of executable file placed on USB drive.
Further, no information related to previous runs of DCode was found in Amcache.hve file. Also, we could not find any information related to applications when the applications 
are run from network drives.

\subsubsection{B4: Installing a Metro App from Windows Store}

This experiment was performed to investigate the effects of installing a Metro App from Windows Store on Amcache.hve. For this, a laptop running Windows 10 Pro was considered and Facebook App was installed from Windows Store. Subsequently, Amcache.hve file was extracted and parsed to observe the artifacts related to Facebook App. It was found that no artifacts related to Metro Apps is recorded in the Amcache.hve file; however, information related to App under consideration were found in ApplicationInventory.xml file. Figure 6 shows information related to Facebook App contained in ApplicationInventory.xml file such as program name, publisher name, installation timestamp etc. As discussed in Section 3, these inventory files are not permanently stored on disk volume. Also, the traces of evidence of Metro Apps were not always found in this inventory file.

\subsection{Set C: Performing Anti-Forensic Attempts on Amcache.hve}

This experiment was conducted to simulate anti-forensic attempts carried out on Amcache.hve file to mislead a forensic investigator. For this, Amcache.hve and associated inventory files were deleted from a system running Windows 10 Pro. It was observed that users cannot delete Amcache.hve file while Windows is running as this file is open in system; however, deletion can be achieved by mounting Windows system drive from a Live Boot DVD of Ubuntu 14.04 LTS. The findings of this experiment for system under consideration are as follows:

- When the Amcache.hve file was deleted, it was recreated on system reboot with new created and modified timestamps. Furthermore, it was also observed that if any host-based executable was run or any software program was installed, then new Amcache.hve file was created in the same session with new created and modified timestamps. The finding of this experiment supports the theory that Amcache.hve is always open in system whilst the computer system is running; however, the changes in the Amcache.hve are written to disk when a system reboot, shutdown, sleep, or hibernation state is initiated.

- The new Amcache.hve file was found smaller in size (32 KB) than the original Amcache.hve file (64 KB following a clean install).

- The further activities caused the new information to be stored in the newly created Amcache.hve file updating its modified timestamp. For example, activity performed here on system under review was installing Mozilla Firefox web browser from USB thumb drive. Table 6 summarizes the behavior (created and modified timestamps) of new Amcache.hve file with respect to considered activity.

To check the consistency of the findings listed above, the same experiment was replicated on a Windows 8 Pro 64-bit system and it was observed that the results were different, as discussed:

- When the Amcache.hve file was deleted, it was not recreated on system reboot. However, any user activity followed by system reboot resulted in creation of new Amcache.hve file.

- Running a host-based executable or installing an executable produced a new Amcache.hve file with new created and 


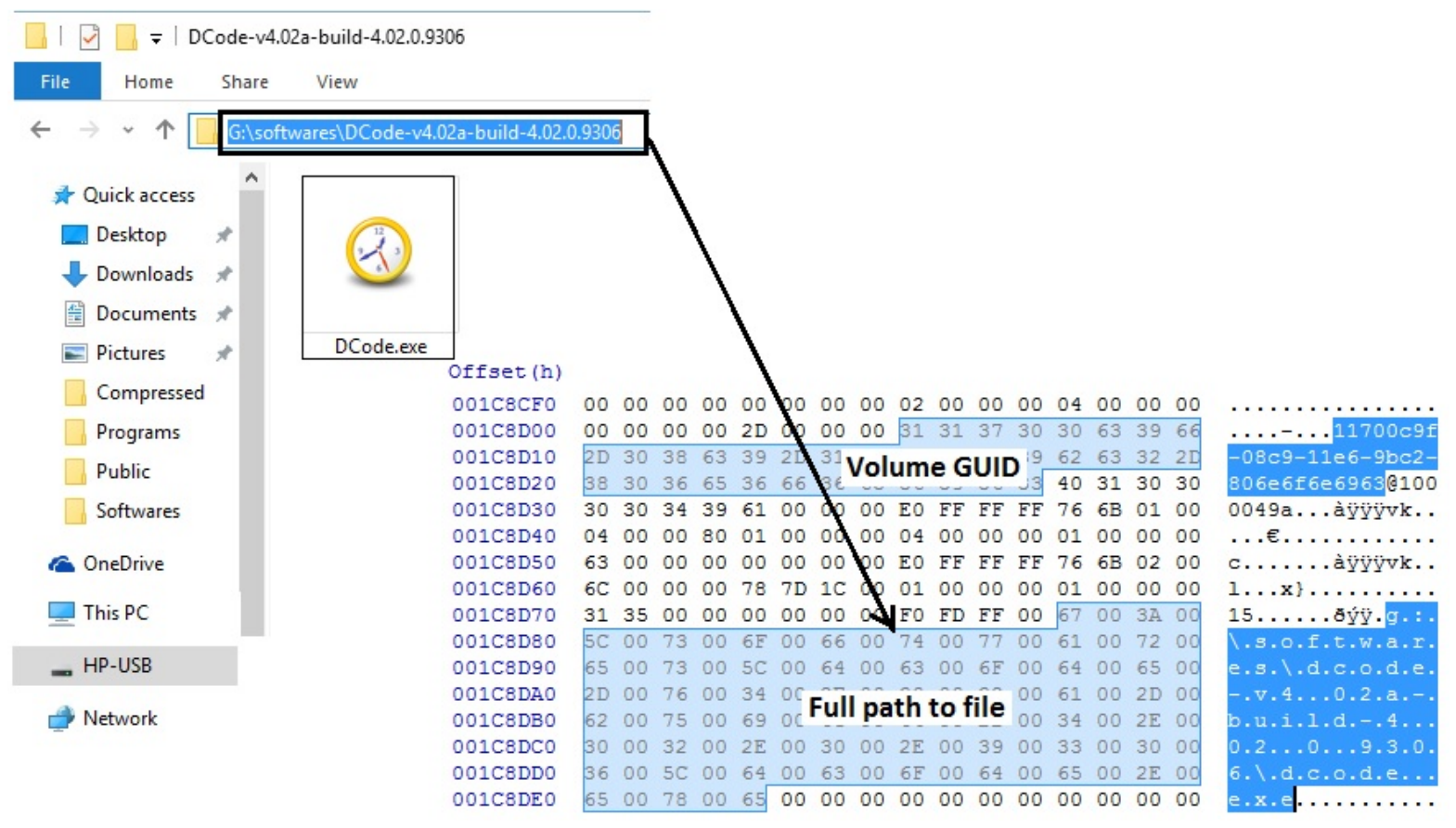

Figure 5. Recorded information related to portable executable in Amcache.hve

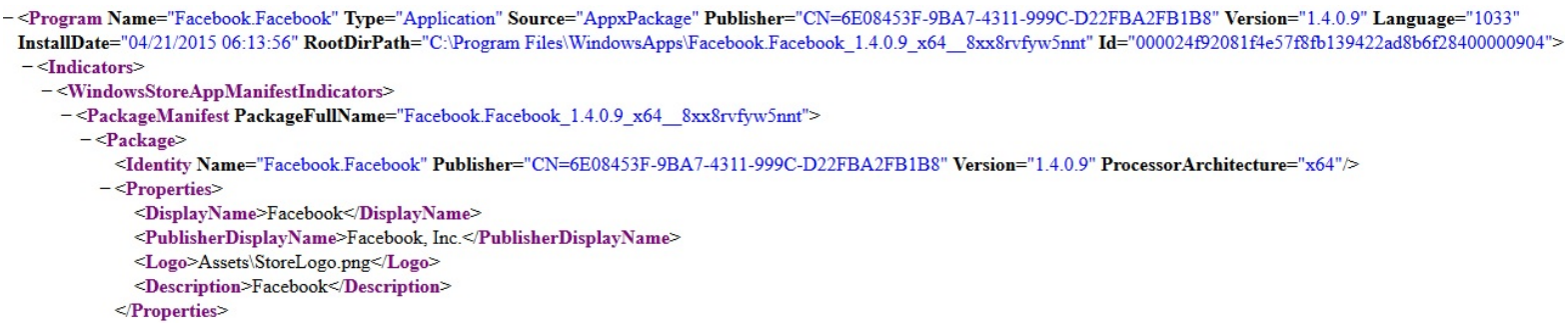

Figure 6. Stored information related to Facebook App in ApplicationInventory.xml file

Table 6. Created and modified timestamps changes to Amcache.hve in Windows 10

\begin{tabular}{|c|c|c|c|c|}
\hline Experiment & Activities & $\begin{array}{l}\text { Size } \\
(\mathrm{KB})\end{array}$ & $\begin{array}{c}\text { Created } \\
\text { timestamp }\end{array}$ & $\begin{array}{l}\text { Modified } \\
\text { timestamp }\end{array}$ \\
\hline A, 1.1 & $\begin{array}{l}\text { Clean install } \\
\text { Amcache.hve }\end{array}$ & 64 & 21/04/16 12:07 & 21/04/16 12:07 \\
\hline A, 1.1 & $\begin{array}{l}\text { Delete Amcache.hve } \\
\text { @12:20, reboot }\end{array}$ & 32 & 21/04/16 12:20 & $21 / 04 / 16 \quad 12: 20$ \\
\hline B3, 1.2 & $\begin{array}{l}\text { Install Mozilla Firefox } \\
\text { visit URLs, reboot }\end{array}$ & 64 & $21 / 04 / 1612: 20$ & $21 / 04 / 1612: 31$ \\
\hline
\end{tabular}

modified timestamps. Size of the new Amcache.hve was observed to be same as of the original. Further, the effect of user activities on Amcache.hve file in
Windows 8 Professional was found to be same as in Windows 10 Pro. 


\subsection{Forensic Utilization of Amcache.hve Information}

The Amcache.hve information can be very useful to track the applications run on a suspected system. The following subsections demonstrate the forensic application of Amcache.hve information.

\subsubsection{Tracking deleted applications}

Previously run applications leave traces in Windows registry, Shortcut files, Jump Lists and in Prefetch folder. However, if the traces from these locations are deleted manually, the information related to run applications can no longer be ascertained; however, the Amcache.hve stores the traces of deleted applications and analyzing it can reveal artifacts of the deleted applications. The analysis can also reveal the timestamp of deletion. Therefore, if the evidence file is deleted, information related to evidence file can be extracted by analyzing the Amcache.hve file.

If a suspected user wipes out all traces of run applications using a privacy protection tool and also cleans the traces of such tools, the investigator cannot find the traces of such anti-forensic behaviors by analyzing the Windows registry, Shortcut files, Jump Lists or Prefetch folder; however, the traces of such privacy protection tools can be obtained by analyzing the Amcache.hve file.

We considered a hypothetical case in which a suspected user wipes out all traces of previously run applications (say, evidence file) by use of a privacy protection tool (CCleaner) followed by the deletion of this tool from the system. The investigator's job is to find out the information related to evidence file and the traces of anti-forensic tool considered in case.

The analysis of Amcache.hve file of the suspected system can reveal the required information to investigator. The investigator can list out all the deleted applications including their execution file path, timestamp created, timestamp of first run, referenced files by applications, timestamp of deletion etc. The Figure 7 shows the traces of CCleaner application in Amcache.hve file when the application has been deleted from the system. The timestamps of applications' installation and deletion can be obtained by decoding Unix 32-bit hex value to date and time format using DCode.

\subsubsection{Tracking malwares}

The Amcache.hve file records hash (SHA-1) of executable file that can provide immensely useful information to track the applications. Malware analysts can leverage this information to track malwares and their referenced files on a victim system. The traces of run malware persist even if it get deleted or wiped itself from the system.

To test if we can trace a malware, a computer system was infected with a malware sample 'xiaose.exe.' The computer system was restarted and allowed to run malware program followed by shutdown to extract Amcache.hve from Windows system using Live Boot DVD of Ubuntu 14.04 LTS. The extracted Amcache.hve was then parsed using Registry Explorer and traces of malware sample was observed as shown in Figure 8. We found SHA-1 hash of xiaose.exe in value 101 as highlighted in the figure. The hash of the malware was found to be same when it was analyzed on Virus Total where it was detected 54 times as a malicious program out of 56 anti-virus. Thus, the hash of executable files can be very useful information to detect the malicious programs run on a system.

\subsubsection{Tracking applications run from external devices}

To identify the portable applications run from external devices is a part of many forensic investigations. The analysis of Amcache.hve file can prove to be a use- 


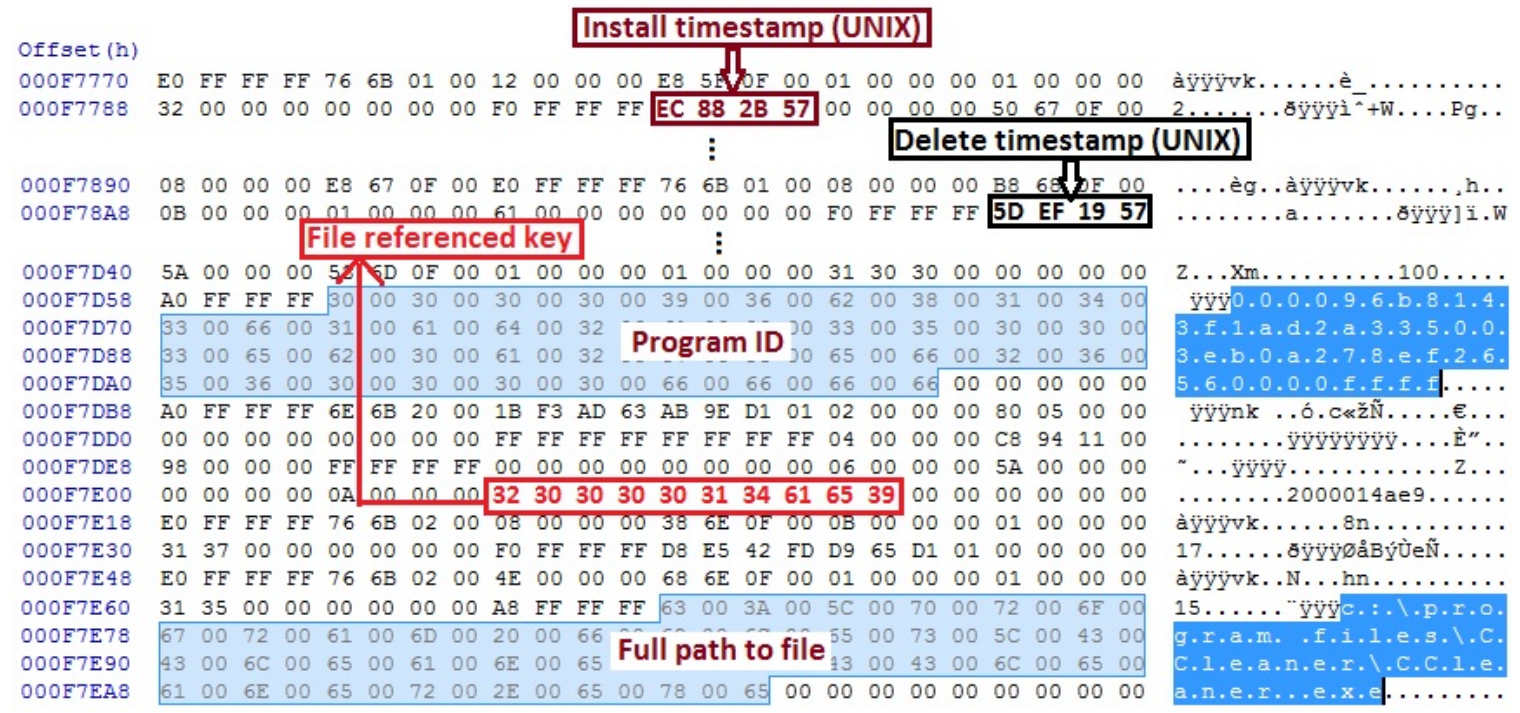

Figure 7. Install and delete timestamps of CCleaner application recorded in Amcache.hve

Parsing Amcache.hve using Registry Explorer

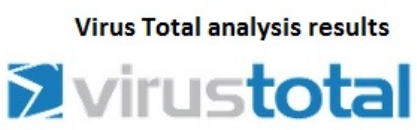

\begin{tabular}{|c|c|}
\hline RegSz & 360安全卫士 主程序模块 \\
\hline RegSz & $360 . \mathrm{cn}$ \\
\hline RegDword & 6 \\
\hline RegSz & $00063513 f 24 a 3938787 a 457 d f 7845 e 3 d 60 c a 00000408$ \\
\hline RegSz & $00006 c 1948 f 7$ edf $115 \mathrm{~cd} 1$ 1f13cd $170 \mathrm{~b} 882077930 \mathrm{be} 150$ \\
\hline RegQword & 130763120360000000 \\
\hline RegQword & 130763120360000000 \\
\hline RegSz & c: |users Jjohn |downloads |compressed/malz5 |xiaose. exe \\
\hline RegDword & 0 \\
\hline RegQword & 130763120360550356 \\
\hline RegSz & $9,8,0,1108$ \\
\hline RegDword & 2052 \\
\hline RegQword & 72057594037928960 \\
\hline RegSz & $9,8,0,1108$ \\
\hline RegDword & 550356 \\
\hline RegDword & 380928 \\
\hline RegSz & 010114ef5688b88453b95b2374996169be039a329ee6 \\
\hline RegDword & 0 \\
\hline RegQword & 2533309150135380 \\
\hline RegQword & 2533309150135380 \\
\hline RegSz & 360安全卫士 主程序模块 \\
\hline RegDword & 0 \\
\hline RegDword & 1259933759 \\
\hline
\end{tabular}

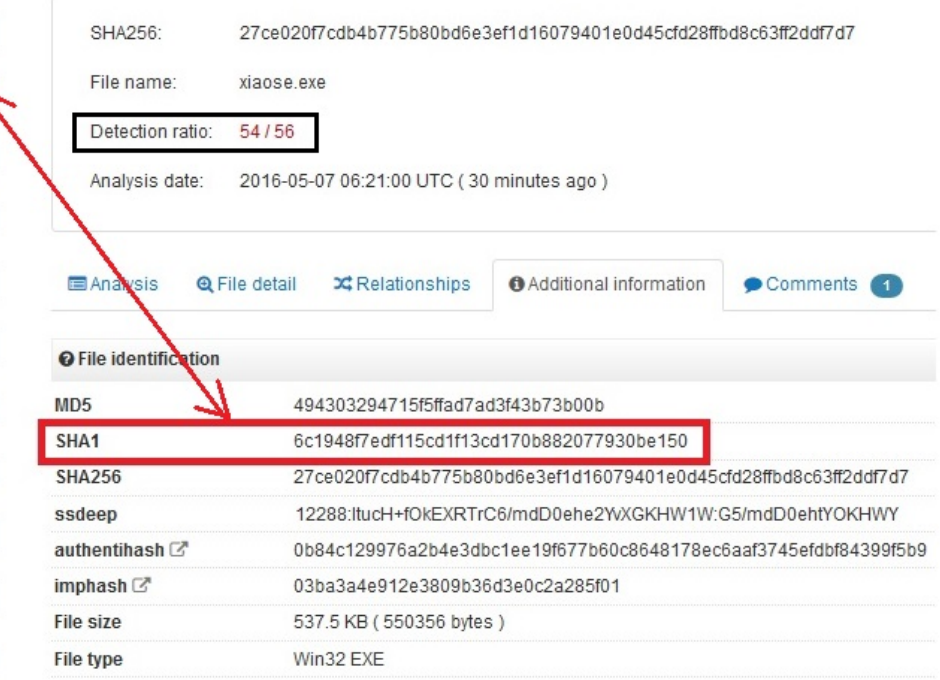

Figure 8. SHA-1 hash of malware program recorded in Amcache.hve

ful forensic utility in the cases where artifacts of external devices mounted on a system have been removed from the UserAssist and MountedDevices registry key. The Amcache.hve file records the full path to executable file from which the application was executed. The drive letter recorded in this file path can distinguish the fixed and re- movable devices. In addition to this, the Volume GUID of external device is also recorded in Amcache.hve file. The experimental results discussed in 4.2.3 and shown in Figure 5 that shows the drive letter and the Volume GUID assigned to a USB device from which the portable executable (DCode) was run. However, no information related to drive la- 
bel and run count of portable executable was observed in Amcache.hve file.

\section{DISCUSSIONS}

This section describes the information that can and cannot be gathered from Amcache.hve file and similar sources. We compare forensic potential of similar sources, namely, IconCache.db, SRUDB.dat (present in Windows 8 and higher versions) and Prefetch folder with Amcache.hve file as shown in Table 7 . The IconCache.db file records the file paths of applications that have been executed, viewed, stored, installed or copied (Lee \& Lee, 2014); however, IconCache.db file does not record any information related to timestamps of the applications. The SRUDB.dat file stores process details including run timestamp, file path and run count of applications; however, the precise startup times are not available from SRUDB.dat file, but it is possible to determine that the application was run on a particular date (Khatri, 2015). Also, the traces from some of deleted applications were not always recorded in SRUM data. Prefetch files can provide information such as application name, file path from which the application was run, created and modified timestamps and run count of applications. However, if an application has been deleted from the system, the delete timestamp is not recorded in the Prefetch file. Also, popular privacy protection tools such as CCleaner, BCWipe, CleanAfterMe or Privacy Eraser are able to delete artifacts in Prefetch files. Fortunately, the Amcache.hve stored artifacts such as the timestamp of an application's first run and traces of deleted applications including the timestamp of deletion, which are not recorded in any other sources considered; however, the information related to run count of applications is not observed in Amcache.hve file. Thus, for better tracking of applications we can correlate and combine the information in IconCache.db, SRUDB.dat and Prefetch files with the information in Amcache.hve file.

\section{CONCLUSION}

User activity analysis on a suspected system is part of many forensic investigations. The Amcache.hve file as a new artifact was first introduced in Windows 8 that records the information related to Windows Application Experience and Compatibility feature. Forensic investigators can leverage the information retained in Amcache.hve file to trace the user activities performed on a system specially related to program executions.

This paper investigates the evidential potential of Windows Amcache.hve file and its application in digital forensics. For identifying the artifacts retained in Amcache.hve and investigating the effects of user activities on Amcache.hve, the experiments were devised in three sets. The results of experiments conducted in this research highlight the important advantages of examining the Amcache.hve file which are as follows:

- It is useful to find recent run applications and their respective history.

- It represents the information related to Windows Application Experience and Compatibility feature.

- The information remains in the Amcache.hve file even when the application and Prefetch folder have been deleted.

- Anti-forensic tools such as CCleaner are not able to remove Amcache.hve file as it is being used during the Windows runtime;

- Amcache.hve file is an excellent source for malware analysts to trace program executions. 
Table 7. Comparison of information recorded in IconCache.db, SRUDB.dat, Prefetch folder and Amcache.hve

\begin{tabular}{|c|c|c|c|c|c|c|c|c|}
\hline & $\begin{array}{l}\text { Application name } \\
\text { and file path }\end{array}$ & $\begin{array}{l}\text { Created } \\
\text { timestamp }\end{array}$ & $\begin{array}{l}\text { Last modified } \\
\text { timestamps }\end{array}$ & $\begin{array}{l}\text { Run } \\
\text { count }\end{array}$ & $\begin{array}{l}\text { Uninstall } \\
\text { timestamp }\end{array}$ & $\begin{array}{l}\text { Referenced } \\
\text { files }\end{array}$ & $\begin{array}{l}\text { Information related } \\
\text { to Metro Apps }\end{array}$ & $\begin{array}{l}\text { Unknown by popular } \\
\text { privacy protection tools }\end{array}$ \\
\hline IconCache.db & $\checkmark$ & $\times$ & $\times$ & $\times$ & $\times$ & $x$ & $\times$ & $\times$ \\
\hline SRUDB.dat & $\checkmark$ & $\checkmark$ & $\times$ & $\checkmark$ & $\times$ & $\times$ & $\checkmark$ & $\checkmark$ \\
\hline Prefetch & $\checkmark$ & $\checkmark$ & $\checkmark$ & $\checkmark$ & $\times$ & $\checkmark$ & $\times$ & $\times$ \\
\hline Amcache.hve & $\checkmark$ & $\checkmark$ & $\checkmark$ & $x$ & $\checkmark$ & $\checkmark$ & $\times$ & $\checkmark$ \\
\hline
\end{tabular}

It was observed that the Amcache.hve file does not record the run count of applications, so forensic investigators are suggested to combine and correlate the artifacts from other sources such as Windows Shortcut files, UserAssist key and Prefetch folder, for constructing comprehensive activity timelines.

\section{FURTHER RESEARCH SCOPE}

The further study on Amcache.hve may be focused on identification of unknown fields in File and Programs keys in Amcache.hve file. The research can also be focused on the analysis of Amcache.hve file when the applications are run, installed or executed from the network drive, Internet or from Windows Store.

\section{ACKNOWLEDGMENTS}

The views and opinions expressed in this article are those of the authors alone. We would like to thank Pankaj Choudhary and Nitesh K. Bharadwaj working in Digital Forensics laboratory of DIAT (DU), Pune and the anonymous reviewers for their assistance and providing constructive and generous comments. Despite their invaluable assistance, any errors remaining in this article are solely attributed to the authors. 


\section{REFERENCES}

AccessData. (2014). Registry viewer. http://accessdata.com/product -download/digital-forensics/ registry-viewer-1-8-0-5. ([accessed 26-Feb-2016])

Carvey, H. (2005). The windows registry as a forensic resource. Digital Investigation, 2(3), 201-205.

Carvey, H. (2011). Windows registry forensics: Advanced digital forensic analysis of the windows registry. Elsevier.

Carvey, H. (2013). Regripper. https://code.google.com/archive/p/ regripper/downloads. ([accessed 26-Feb-2016])

Carvey, H., \& Altheide, C. (2005). Tracking usb storage: Analysis of windows artifacts generated by usb storage devices. Digital Investigation, 2(2), 94-100.

Collie, J. (2013). The windows iconcache.db: A resource for forensic artifacts from usb connectable devices. Digital Investigation, 9(3), 200-210.

Davis, A. (2012). Leveraging the application compatibility cache in forensic investigations. http://dl . mandiant.com/EE/library/ Whitepaper_ShimCacheParser.pdf. ([accessed 21-March-2016])

Harrell, C. (2013). Revealing the recentfilecache.bcf file.

http://journeyintoir.blogspot.in/ 2013/12/revealing -recentfilecachebcf-file.html. ([accessed 14-April-2016])
Khatri, Y. (2013). Amcache.hve in windows 8 - goldmine for malware hunters. http: //www. swiftforensics.com/ 2013/12/amcachehve-in-windows-8 -goldmine-for.html. ([accessed 10-March-2016])

Khatri, Y. (2015). Forensic implications of system resource usage monitor (srum) data in windows 8. Digital Investigation, 12, 53-65.

Kim, M., \& Lee, S. (2015). Forensic analysis using amcache.hve. In Digital forensics and cyber crime: 7th international conference, icdf2c 2015, seoul, south korea, october 6-8, 2015. revised selected papers (Vol. 157, p. 215).

Lee, C.-Y., \& Lee, S. (2014). Structure and application of iconcache.db files for digital forensics. Digital Investigation, $11(2), 102-110$.

Mee, V., \& Jones, A. (2005). The windows operating system registry-a central repository of evidence. In Proceedings from e-crime and computer evidence conference (Vol. 2005).

Mee, V., Tryfonas, T., \& Sutherland, I. (2006). The windows registry as a forensic artefact: Illustrating evidence collection for internet usage. digital investigation, 3(3), 166-173.

Microsoft. (2016). Understanding shims. https://technet.microsoft.com/en us/library $/$ dd $837644 \% 28 v=w s .10 \% 29$. aspx. ([accessed 09-March-2016])

NirSoft. (2013). Regscanner. http://www . nirsoft.net/utils/ regscanner.html. ([accessed 26-Feb-2016]) 
Singh, B., \& Singh, U. (2016). A forensic insight into windows 10 jump lists. Digital Investigation, 17, 1-13.

Singh, B., \& Singh, U. (2017). A forensic insight into windows 10 cortana search. Computers \&5 Security, 66, 142-154.

Wong, L. W. (2007). Forensic analysis of the windows registry. Forensic Focus, 1.

Zimmerman, E. (2015). Registry explorer/recmd version 0.7.1.0. https://ericzimmerman.github.io/. ([accessed 26-Feb-2016]) 